\title{
Application of Mathematical Morphology in GIS PD Signal Analysis
}

\author{
Qi Liu ${ }^{1}$ and Qiang $\mathrm{Gao}^{2, *}$ \\ ${ }^{1}$ State Grid Liaoning Electric Power Company Electric Power Research Institute, Shenyang, China \\ ${ }^{2}$ Shanghai Jiao Tong University, Shanghai, China \\ ${ }^{*}$ Corresponding author
}

\begin{abstract}
In order to solve the problem that with the interference of on-site GIS to PD signals, the type diagnosis and fault location of insulation defect are affected. This paper proposed a new morphological filtering algorithm based on morphological filtering theory is proposed, which combines the basic operations of erosion, dilation, morphological open and morphological close and constructed a new combined morphological filter algorithm for GIS signal de-noising. Through filtering the simulated noised PD pulse, the results showed that morphological filtering can not only remove white noise effectively, but also suppress the narrowband interference effectively. Three sets of evaluation indexes are introduced and compared with the wavelet de-noising method, and the effectiveness of morphological filtering in suppressing noise and extracting PD pulse is verified.
\end{abstract}

Keywords-GIS; PD; morphological; de-nosing

\section{INTRODUCTION}

PD detection is an important research topic in the insulation diagnosis of power equipment. PD is not only a sign of insulation aging of power equipment, but also the main reason for further deterioration of insulation. Through PD detection, the insulation condition of equipment can be effectively grasped to avoid the occurrence of failure. However, there is always a strong external interference in the field, so that the detected PD signals often contain interference components. Among the interference components, the mixed interference including the periodic interference and white noise is relatively strong, and can even submerge PD signals. In addition, it is very important to locate the discharge source quickly and accurately after discovering the existence of the $\mathrm{PD}$, which is very important for the safe operation of the system.

Mathematical Morphology (MM) ${ }^{[1-3]}$ is a typical non-linear signal processing method developed in recent years. The theory was founded in the mid-1960s by Matheron and Serra, mathematicians at the Paris School of Mines. MM has some obvious advantages over other time-domain or frequencydomain image processing and analysis methods. It can effectively filter out noise with morphological operators while preserving the original information. In the various operations in MM, the choice of structural elements is very essential to the ability to effectively suppress the noise and extract the signal. Morphology has been widely used in image signal processing, medical imaging diagnosis and medical magnetic resonance and mechanical vibration signal noise reduction and other fields. With the help of the above research, scholars at home and abroad have begun to apply MM to signal analysis of power systems. The main applications are using MM to denoise the MOA leakage current monitoring signals, using MM to detect the mutation signal of power systems, using MM and the short window power algorithm to detect and locate the power quality disturbances while eliminating random and impulse noises, using MM to solve the problem of noise pollution in the collected on-site data and eliminate DC bias, applying MM to the study of harmonic detection of power system and the combination of wavelet transform, fractal theory and neural network, using MM to de-noise the PD signals. These applications have all achieved good results.

Based on the above research, this paper constructs a new cascaded morphological filtering method to de-noise and filter the PD signal of GIS.

\section{MM FILTERING PRINCIPLE}

$\mathrm{MM}$ is used as a nonlinear filtering technique. It uses the waveform information of the signal to design a "probe" called a structural element. By continuously moving the probe in the signal, useful information can be extracted for the entire signal waveform to achieve the purpose of extracting the signal, preserving details and suppressing the noises . Morphological transformation is generally divided into binary transformation and multivalued transformation. Multivalued transformation is also called grayscale transformation. Since PD signals are onedimensional signals, this chapter only discusses the multivalued morphological transformations in the case of onedimensional discrete signals, including erosion, dilation, morphological open and morphological close, as well as the combined filter cascaded by morphological open and morphological close.

\section{A. Erosion and Dilation}

Erosion and dilation are two basic MM operations. Dilation is the dual operation of erosion, which can be defined as the erosion calculation of the complement of the image. They are defined as follows:

1) Erosion: Let the original signal $f(n)$ be a discrete function defined on $F=\{0,1, \ldots, N-1\}$. The structural element $k(n)$ is a discrete function defined on $K=\{0,1, \ldots, M-1\}$, where $N>M$. 
The function $f$ is eroded by the structural element $k$ as:

$$
(f \Theta k)(m)=\min _{\mathrm{m}=0, \cdots, \mathrm{N}-\mathrm{M}} f(m+n)-k(n), \mu=0,1, \ldots, \mathrm{N}-\mathrm{M}
$$

Erosion is a shrink operation to the signal, so the signal value $f \Theta k$ after erosion is always less than the original signal $f$. The erosion value $(f \Theta k)(m)$ of the signal $f$ at point $m$ can be obtained by the following procedure:

a) Determining the structural element values at point $m$.

b) The original signal minus the structural element value.

c) Take the minimum value of each value.

2) Dilation: The function $f$ is dilated by the structural element $k$ as:

$$
(f \oplus k)(m)=\max _{n=m-M+1, \ldots, m} f(n)+k(m-n), \mu=\mathrm{M}-1, \mathrm{M}, \ldots, \mathrm{N}-1
$$

Dilation is an expansion operation to the signal, so the signal value $f \oplus k$ after dilation is always greater than the original signal $f$. The dilation value $(f \oplus k)(m)$ of the signal $f$ at point $m$ can be obtained by the following procedure:

a) Determining the structural element values at point $m$ after negation.

b) Add the original signal and the value of the structure element.

c) Take the maximum value of each value.

\section{B. Morphological Open and Morphological Close}

In the morphological treatment, in addition to the two basic operations, there are morphological open and close operations cascaded by erosion and dilation. These two operations are the most important operations, or transformations, in mathematical morphology. Morphological open is defined as the signal is first eroded and then dilated. Morphological close is defined as the signal is first dilated and then eroded ${ }^{[4-6]}$.

The morphological open and close operations of $f(n)$ with respect to $k(n)$ are defined as:

$$
\begin{aligned}
& (f \circ k)(n)=(f \Theta k \oplus k)(n) \\
& (f \bullet k)(n)=(f \oplus k \Theta k)(n)
\end{aligned}
$$

Where $\circ$ and $\cdot$ respectively represent morphological open and morphological close. The open and closed states have a smoothing function. Open operation can suppress the peak noise in the signal, "cut" the peak. Closed operation can remove the low noise in the signal, "fill" the trough.
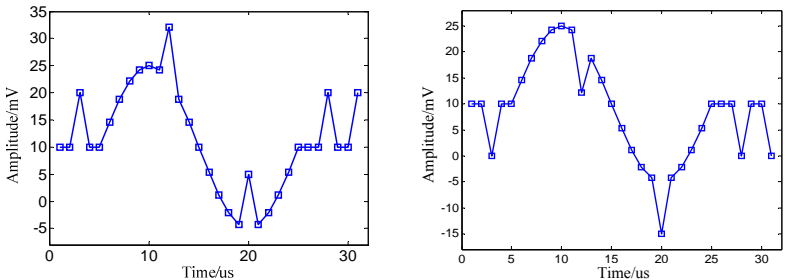

(a) Simulation signal
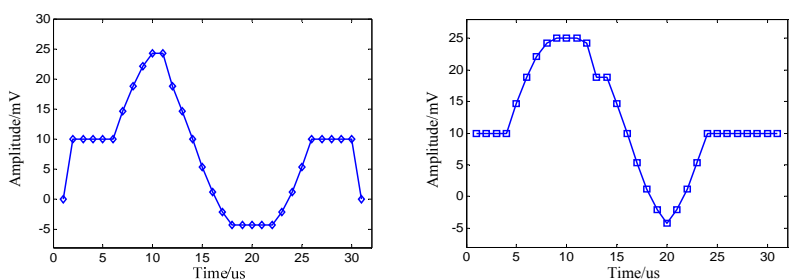

(b) Erosion result
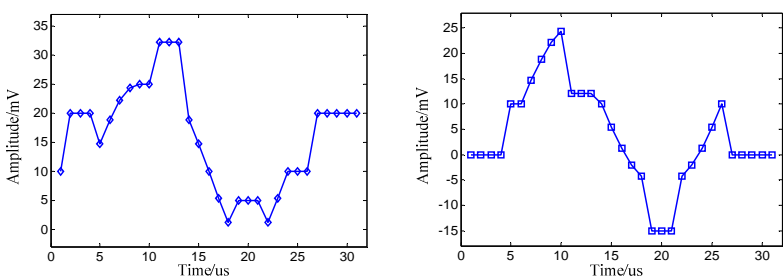

(c) Dilation result
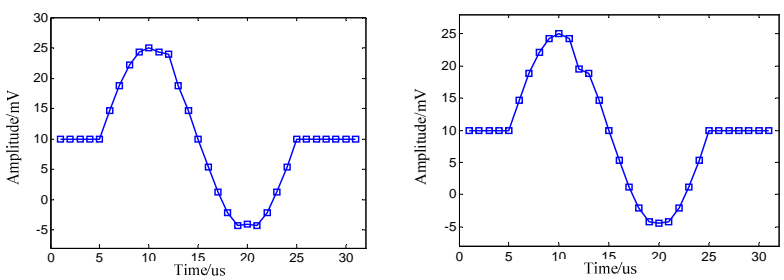

(d) Morphological open filtering result

FIGURE I. FILTERING RESULTS OF ERODE, DILATION AND OPEN FOR SIGNAL WITH POSITIVE AND NEGATIVE PULSE INTERFERENCES

The open operation on the data is equivalent to moving the structure element down the signal. The result of the open operation is the maximum value of the structural element at that point. Similarly, the closing operation is equivalent to the structural elements in the data above the move. The result of the close operation is the minimum value of the structural element at that point.

The results of open and close operations are affected by the shape of the structure element. The shape of the structure element should be designed specifically according to different signal waveforms which required processing. For example, after using a flat structure element of length $M$ to process the open operation, the original signal no longer contains the peak whose length is less than $M-1$ (positive pulse interference). Similarly, after using the structure element to process the close operation, the original signal no longer contains the trough 
whose length is less than $M-1$ (negative pulse interference). Then positive and negative pulse interference are removed.

\section{Selection of Structural Elements}

The role of structural elements in morphological operation is equivalent to a filter window. Its shape and size will have a direct impact on the result of operation. In the process of noise suppression, the integrity of the signal should be ensured, that is structural elements should be designed according to the different characteristics of the signal and noise waveform information. Structure elements have three key factors: shape, width and height. The shape should be determined from the original signal waveform. The waveform features of the selected structural element should be as similar as possible to the PD signal waveforms to reduce distortion of the signal. The main shapes are linear, curve (quadratic, cubic, etc.), triangular, circular and polygonal. The width and height are determined by the single pulse time width and signal sample rate in the signal.

Figure I (a) shows the simulation contains a positive (peak) and negative (trough) pulses interference sine wave signal. (b), (c) and (d) respectively show the signal waveform after the process of erosion, dilation and morphological open operation. As the figures show, the result of suppressing the spike by using the erosion or dilation operation alone is not satisfactory while morphological open operation can filter out spikes better. However, the open operation results in a slight distortion near the positive and negative half-cycle peak in the original pulse, another result of suppressing the spike by using the dilation or erosion operation alone is not satisfactory while morphological close operation can filter out spikes better. However, similar to the open operation, the close operation also results in a slight distortion near the positive and negative half-cycle peak in the original pulse.

In order to solve the above problems, a new combined morphological method is proposed to synthesize the filter results by cascading the morphological open and close filters. In the filtering process, through optimizing the structural elements, the method can overcome the above shortcomings and obtain better filtering results.

\section{CONSTRUCTION OF NEW MORPHOLOGICAL FILTERING ALGORITHM}

For the white noise and narrow-band interference signals contained in the PD signal, a new cascaded filter algorithm is proposed in this section based on the morphological open filter and the morphological closed filter. The function of the algorithm includes two parts: suppressing noise and eliminating narrow-band interference signal. The block diagram of the algorithm is shown in Figure II

In the noise suppression section (A shown in Figure II), positive and negative pulsed interference signals including white noise in the signal can be suppressed by two groups of cascaded open / close filters. In order to improve the precision of the algorithm, two sets of filter channels are constructed. One group of signals goes through the open operation first and then through the close operation. The other group of signals goes through the close operation first and then through the open operation. During the process of the two groups of channel, select the same structural elements and average the processing results of two groups of channel. The resulting signal is represented by $S_{1} . S_{1}$ is subtracted from the original signal to obtain the background noise in the original signal.

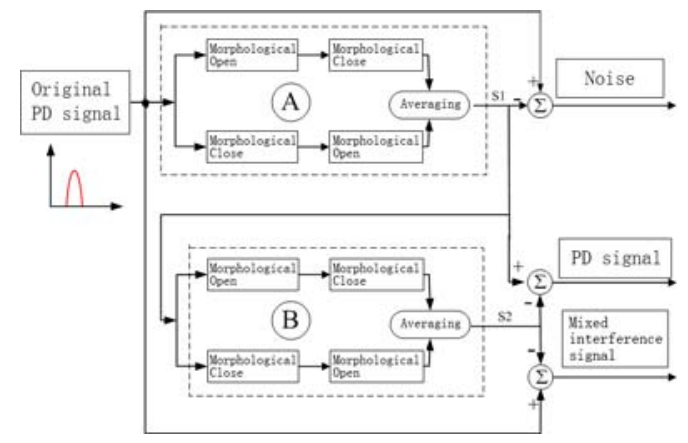

FIGURE II. DIAGRAM OF NEW MORPHOLOGICAL FILTER

It should be noted that in the suppression of narrowband interference part of the first group of channel processing, two different structural elements need to be designed to eliminate the noise generated by the suppression of crests and troughs. The structural elements are also determined by PD pulses. The pulse duration is $\mathrm{T}$ seconds and the sampling rate is $\mathrm{S} \mathrm{Hz}$, then the pulse width $\mathrm{T} \times \mathrm{S}$. Test results show that the open structure length $\mathrm{L}$ must be much larger than $\mathrm{T} \times \mathrm{S}$, while the length of the closing operation structure element for eliminating the trough can be taken as $2 \mathrm{~L}$.

\section{SimUlation ANALYSIS}

In contrast to narrowband interference, PD signals are nonstationary, non-linear signals with oscillatory attenuation. When a PD pulse is generated inside the power plant and propagates from the discharge source to the sensor, a large attenuation and oscillation are generated due to the capacitive and inductive effects of the sensing path. Therefore, SingleExponential Attenuating and Oscillating Waveform (SEAOW) and Double-Exponential Attenuation and Oscillation Waveform (DEAOW) can be used in the simulation of PD signals. In order to verify the effect of the designed morphological filter, two sets of single-exponential oscillation decay model and two sets of double-exponential oscillation decay model were selected as the simulation PD signals. In order to verify the effect of the designed morphological filter, two sets of single-exponential decay model and two sets of double-exponential decay models were selected to simulate the PD. Simulation parameters are shown in Table I. The data sampling rate is $20 \mathrm{MHz}$.

TABLE I. MODEL PARAMETERS OF SIMULATED PD SIGNALS

\begin{tabular}{|c|l|c|c|c|}
\hline model & $\begin{array}{c}\text { Amplitude } \\
/ \mathbf{m V}\end{array}$ & $\begin{array}{c}\text { Attenuation } \\
\text { coefficient /us }\end{array}$ & $\begin{array}{c}\text { frequency } \\
\text { /MHz }\end{array}$ \\
\hline 1 & SEAOW & 50 & 8 & 0.2 \\
\hline 2 & SEAOW & 60 & 8 & 0.2 \\
\hline 3 & DEAOW & 70 & 9 & 0.25 \\
\hline 4 & DEAOW & 80 & 9 & 0.25 \\
\hline
\end{tabular}

In order to verify the effect of cascaded morphological filtering algorithm, the wavelet with the generating function of 
$\mathrm{db} 8$ is selected, and morphological filter and wavelet filter are applied to PD pulse containing white noise and narrowband periodic interference.
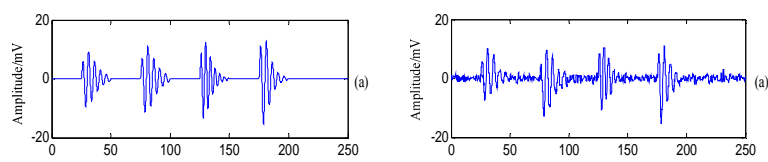

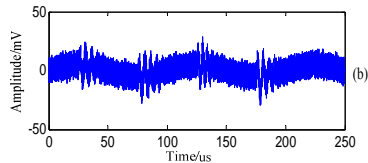

(a)

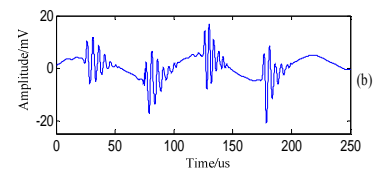

(b)
FIGURE III. (A)PD PULSE OF SIMULATED AND ADDED NOISES (B)SIGNALS AFTER MM AND WAVELET FILTERS

Figure III (a) is the result of morphological filtering. As can be seen, the narrow-band periodic interference and most of the white noise interference have been removed. The oscillations and decay of the rising and falling edges of the extracted PD signals are very close to the original PD pulse. Figure III (b) shows the results of wavelet de-noising. It can be seen that although the wavelet has a very strong suppression effect on the white noise, it is not effective to suppress the narrow-band periodical interference.

\section{TABLE II. COMPARISON RESULTS OF MORPHOLOGICAL AND} WAVELET

\begin{tabular}{|l|c|c|c|}
\hline \multicolumn{1}{|c|}{ method } & $\mathbf{d}_{\mathbf{1}} \mathbf{( \% )}$ & $\mathbf{d}_{\mathbf{2}}(\mathbf{\%})$ & $\mathbf{d}_{\mathbf{3}}(\mathbf{\%})$ \\
\hline Morphological filtering & 6.2 & 3.1 & 19.8 \\
\hline Wavelets & 10.4 & 6.3 & 25.1 \\
\hline
\end{tabular}

To further quantify the effect of morphological filtering, three kinds of evaluation indexes were introduced to analyze the signals before and after filtering. The results are shown in Table II.

$$
\begin{aligned}
& d_{1}\left(s, s^{\prime}\right)=\frac{1}{R} \frac{1}{l} \sum_{n=1}^{l}\left|s(n)-s^{\prime}(n)\right| \\
& d_{2}\left(s, s^{\prime}\right)=\frac{1}{R}\left\{\frac{1}{l} \sum_{n=1}^{l}\left|s(n)-s^{\prime}(n)\right|\right\}^{1 / 2} \\
& d_{3}\left(s, s^{\prime}\right)=\frac{1}{R} \max _{n=1, \cdots, l}\left|s(n)-s^{\prime}(n)\right|
\end{aligned}
$$

Where $s$ and $s^{\prime}$ are respectively the signals before and after the process. Their length is $l . d_{1}$ is the average error between $s$ and $s^{\prime} . d_{2}$ is the root-mean-square deviation between $s$ and $s^{\prime}$. $d_{3}$ is the maximum error between $s$ and $s^{\prime} . R$ is the peak-topeak value of signal $s$ for normalizing $d_{1}, d_{2}$, and $d_{3}$.

As can be seen from Table II, the morphological filtering method is superior to the wavelet de-noising method in terms of the average error $d_{1}$, the root mean square error $d_{2}$ and the maximum error $d_{3}$. At the same time, the validity of the morphological filtering method in PD signal processing is verified.

\section{CONCLUSION}

Based on the theory of mathematical morphological filtering, combining with the basic operations of erosion, dilation, shape opening and shape closure, this paper constructs a new combined morphological filtering algorithm for the de-noising of GIS PD signals.

1) According to the difference of white noise and narrowband interference pulse width, structural elements with different lengths are chosen respectively to suppress the interference signal, and high precision can be obtained. At the same time, in order to achieve a better match with the pulse signal to be extracted, the triangular structure element is selected to process the PD pulse signal to achieve the purpose of suppressing noise and extracting the useful signal characteristics.

2) The results show that morphological filtering can not only remove white noise effectively, but also can suppress the narrow - band interference effectively. Three sets of evaluation indexes are introduced and compared with the wavelet denoising method and the effectiveness of morphological filtering in suppressing noise and extracting PD pulse is verified.

\section{REFERENCES}

[1] Ashtiani, M. B., \& Shahrtash, S. M. (2012). Feature-oriented de-noising of partial discharge signals employing mathematical morphology filters [J]. IEEE Transactions on Dielectrics and Electrical Insulation, 19(6), 2128-2136.

[2] Chan, J. C., Ma, H., Saha, T. K., \& Ekanayake, C. (2014). Self-adaptive partial discharge signal de-noising based on ensemble empirical mode decomposition and automatic morphological thresholding [J]. IEEE Transactions on Dielectrics and Electrical insulation, 21(1), 294-303.

[3] LI, T. Y., YANG, M., ZHOU, X. C., \& CHENG, S. Y. (2007). Method of partial discharge signal analysis based on wavelet transform and mathematical morphology [J]. Power System Technology, 6, 012.

[4] Wangliu, C., Yuexia, G., Jing, W. A. N. G., Tian-yun, L. I., Xin, W. U., \& Jun-jie, W. E. I. (2008). Application of Fast Fourier Transform and Generalized Morphological Filter in Suppression of Narrow-Band Interference in Partial Discharge Signal [J]. POWER SYSTEM TECHNOLOGY-BEIJING-, 32(10), 94.

[5] Ji, T. Y., Tang, W. H., \& Wu, Q. H. (2013). Partial discharge location using a hybrid transformer winding model with morphology-based noise removal.Electric Power Systems Research, 101, 9-16.

[6] LIU, S. B., LV, C., WANG, L. X., \& YU, J. L. (2009). Depression of Narrowband Interference in Partial Discharge Signals of Transformers with Combined Generalized Morphological Filters [J]. High Voltage Engineering, $9,022$. 